\title{
The JLab Eta Factory (JEF) Program in Hall-D at Jefferson Lab
}

\section{S. Taylor ${ }^{* \dagger}$}

Jefferson Lab, USA

E-mail: staylorejlab.org

The JLab Eta Factory (JEF) program aims to use decays of the $\eta$ meson to study higher order terms in Chiral Pertubation theory, to determine the up-down quark mass ratio, and to probe for C-violating/P-conserving processes and other physics beyond the Standard Model. The program will take place at Jefferson Lab using the GlueX detector in Hall D. This article focuses on the rare $\eta \rightarrow \pi^{0} \gamma \gamma$ decay, which requires an upgrade to the existing forward calorimeter in the GlueX detector.

The 9th International workshop on Chiral Dynamics

17-21 September 2018

Durham, NC, USA

* Speaker.

†for the GlueX collaboration 
The $\eta$ meson is one of the Goldstone bosons that arises from spontaneous symmetry breaking of QCD. It has a narrow width $(1.31 \pm 0.05 \mathrm{keV}[1])$ and well-established quantum numbers $I^{G}\left(J^{P C}\right)=0^{+}\left(0^{-+}\right)$. The JLab Eta Factory (JEF) program seeks to leverage $\eta$ decays to probe a wide variety of physical processes, such as

- Constraining the light quark mass ratio.

- Directly constraining new C-violating/Parity-conserving physics.

- Probing the interplay of vector meson dominance and scalar resonances in Chiral Perturbation Theory at $\mathscr{O}\left(p^{6}\right)$.

- Searching for a dark $B$-boson in the channel $B \rightarrow \pi^{0} \gamma$.

The primary focus of the experiment is the rare decay channel $\eta \rightarrow \pi^{0} \gamma \gamma$. This channel allows unique access to higher order terms in Chiral Perturbation theory because the tree level terms at $\mathscr{O}\left(p^{2}\right)$ and $\mathscr{O}\left(p^{4}\right)$ vanish and the first non-zero terms that arise at $\mathscr{O}\left(p^{4}\right)$ involve pion loops that are suppressed due to G-parity or loops involving kaons that are suppressed due to the kaon mass. The first sizable terms contribute at $\mathscr{O}\left(p^{6}\right)$ [2]. The mass distribution of the two photons recoiling against the $\pi^{0}$ contains further information: the shape is sensitive to contributions from scalar resonances as well as vector meson dominance (VMD)[3]. Calculations by Oset, Palaez, and Roca[4] indicate that the presence of chiral loops enhance the two-photon mass distribution at higher masses relative to the flatter distribution arising from VMD, as shown in Fig 1, where the long-dashed curve indicates the tree-level VMD contribution and the solid curve is the full model result .

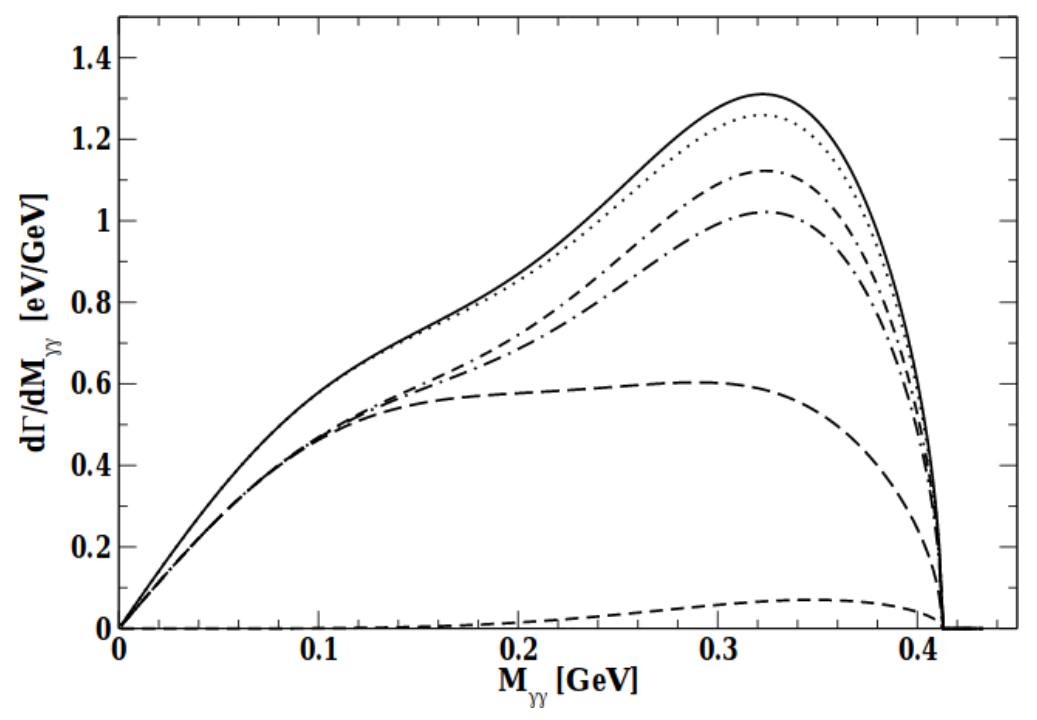

Figure 1: Model prediction for the mass distribution of the two photons recoiling against the $\pi^{0}$ for the $\eta \rightarrow \pi^{0} \gamma \gamma$ channel. Figure taken from [4].

The $\eta \rightarrow \pi^{0} \gamma \gamma$ decay can also be used to search for the signature of dark matter interactions within a particular model. Dark matter accounts for most of the matter in the universe but is 
poorly understood. A natural extension to the Standard Model that could accommodate dark matter interactions is to introduce a new $U(1)_{B}$ gauge symmetry that leads to a new gauge $B$-boson that couples predominantly to quarks. Allowing for kinetic mixing with Standard Model fields, the QCD Lagrangian acquires the following additional terms [9]:

$$
\mathscr{L}=\left(\frac{1}{3} g_{B}+\varepsilon Q_{q} e\right) \bar{q} \gamma^{\mu} q B_{\mu}-\varepsilon e \bar{l} \gamma^{\mu} l B_{\mu},
$$

where the first term shows the explicit coupling to quark fields $(q)$ and the second term allows for kinetic mixing with lepton fields $(l)$. The current limits in the mass/coupling constant parameter space are shown in Fig. 2.

Tulin[9] computed the branches for the $B$ to decay to various combinations of standard model particles, as show in Fig. 3. In the $150-600 \mathrm{MeV}$ mass range the $B$-boson prefers to manifest itself in the $\pi^{0} \gamma$ channel, suggesting a search in the decay $\eta \rightarrow \gamma B \rightarrow \gamma\left(\pi^{0} \gamma\right)$ for which the rare decay discussed above is an irreducible Standard Model background.

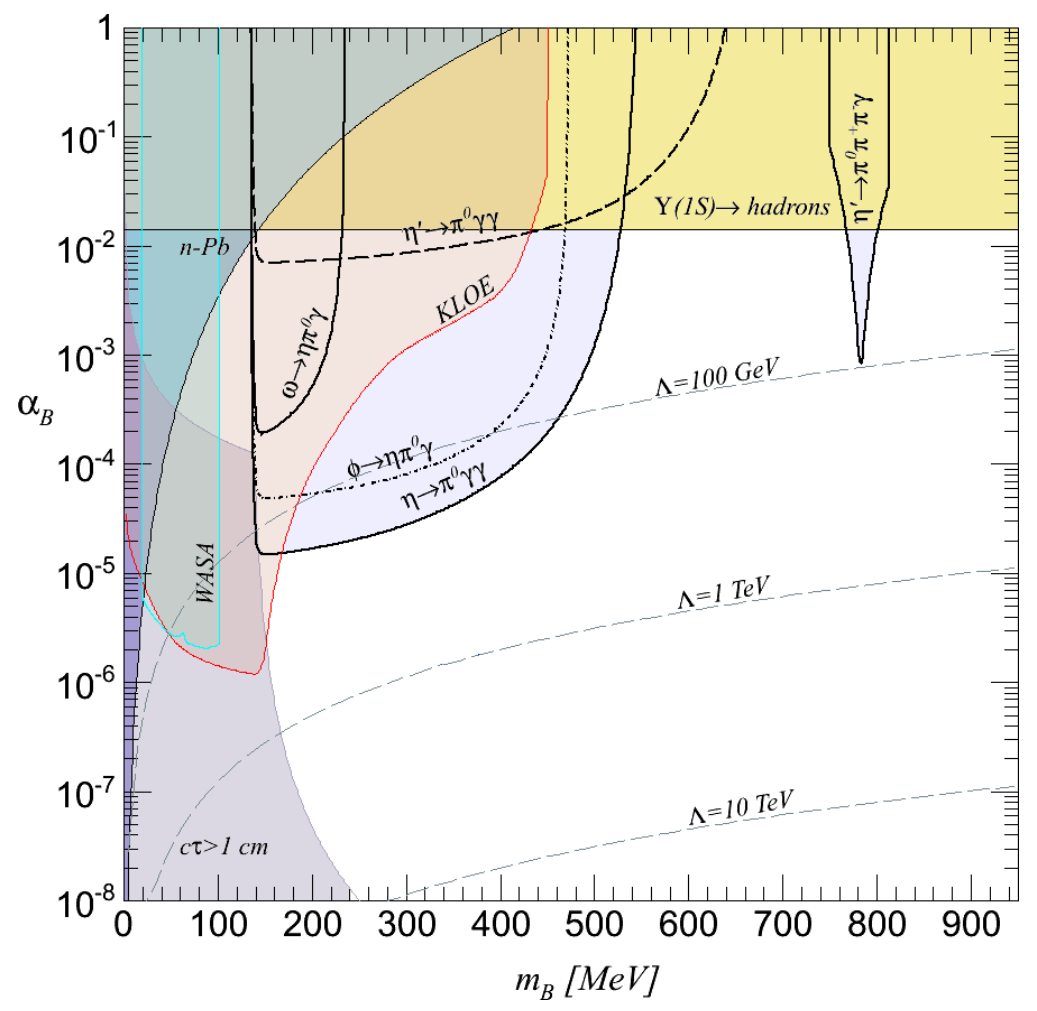

Figure 2: Excluded $B$-boson parameter space adapted from [9]. Here $\alpha_{B} \equiv g_{B}^{2} /(4 \pi)$.

This channel is difficult to extract experimentally. GAMS-2000[5] saw a clear peak for the signal (see Fig. 4) but suffered from low statistics; the result for the partial width, $\Gamma=0.84 \pm$ $0.18 \mathrm{eV}$, is significantly higher than the PDG fitted value of $\Gamma=0.334 \pm 0.028 \mathrm{eV}$ [1]. More recent experiments $[6,7]$ fought large backgrounds under the peak at the $\eta$ mass that were mostly due to $\eta \rightarrow 3 \pi^{0}$ leaking into the signal region due to missing photons or merged clusters; sample plots in 


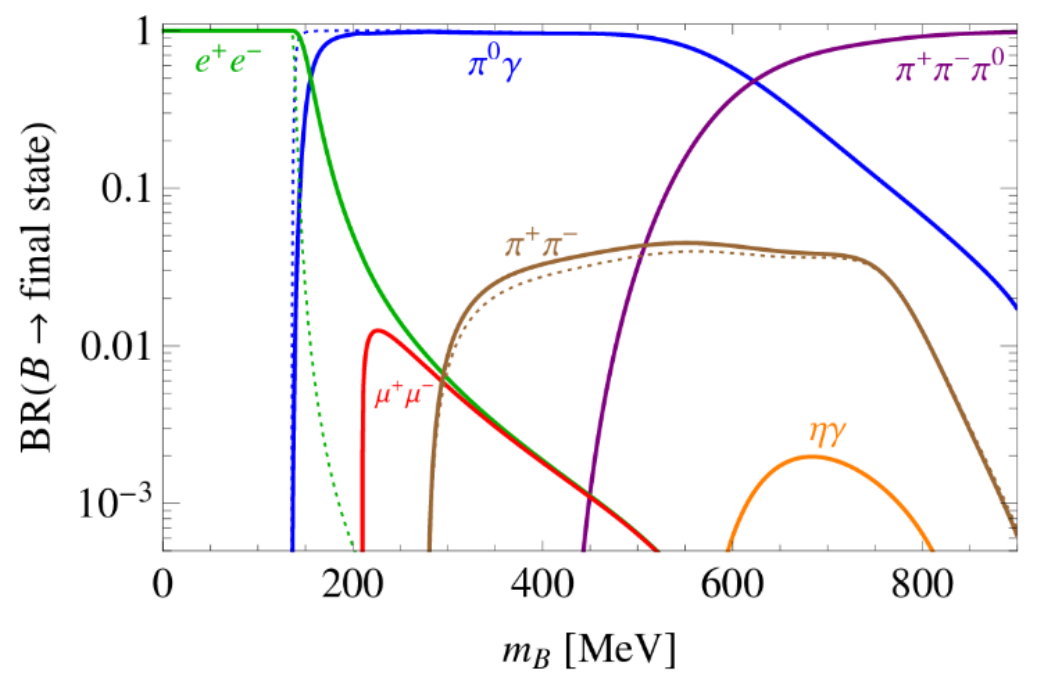

Figure 3: Branching ratios for various decay channels of the $B$-boson as a function of mass. Figure taken from [9].

Fig. 4 from the Crystal Ball experiment at AGS[6] and the MAMI A2 experiment [7] illustrate the problem.

The JEF program takes advantage of the existing GlueX detector housed in Hall-D at Jefferson Lab (JLab). The $12 \mathrm{GeV}$ electron beam from the CEBAF racetrack is directed toward the Tagger hall in the Hall-D complex where it is converted to a photon beam via the insertion of a diamond radiator (see Fig. 5). The photon beam impinges on a liquid hydrogen target at the center of the GlueX spectrometer, shown in Fig. 6. Charged particles emerging from the target region after interactions of the beam with the target protons are detected by two sets of drift chambers: the Central Drift Chambers (CDC) consist of rings of straw tubes that allow tracking for larger angles $\left(>\sim 20^{\circ}\right)$ relative to the beam line and the Forward Drift Chambers (FDC) consist of planes of cathode strip chambers for tracking forward-going particles. Low-momentum protons are separated from lighter particles by computing $d E / d x$ in the CDC. Neutral particles are detected with the Barrel Calorimeter, consisting of layers of lead interleaved with scintillating fibers, and the Forward Calorimeter (FCAL), an array of lead glass blocks. Precise timing information is provided by the Start Counter, a set of 30 thin scintillators surrounding the target region, and the Time-of-Flight, two walls of scintillator paddles immediately upstream of the FCAL.

Data with the GlueX detector were taken in spring 2016 and spring 2017. Clear $\pi^{0}$ peaks were reconstructed for pairs of clusters in the BCAL and the FCAL (Fig. 7) and well-established scalar and tensor mesons were observed in $\pi^{0} \pi^{0}$ and $\pi^{0} \eta$ invariant mass distributions (Fig. 8); however, preliminary investigations suggest that the resolution of the existing Forward Calorimeter is not sufficient to isolate the rare $\eta \rightarrow \pi^{0} \gamma \gamma$ channel from the dominant background from $\eta \rightarrow 3 \pi^{0}$. The measured $\pi^{0} \gamma \gamma$ mass distribution is shown in Fig. 9.

The JEF program calls for replacing the inner blocks of the FCAL with an insert consisting of an array of $2 \times 2 \times 18 \mathrm{~cm}^{3} \mathrm{PbWO}_{4}$ crystals, as illustrated in Fig. 10 for a $100 \times 100 \mathrm{~cm}^{2}$ insert. The insert region affords a factor of two improvement in both energy resolution and position. Monte 

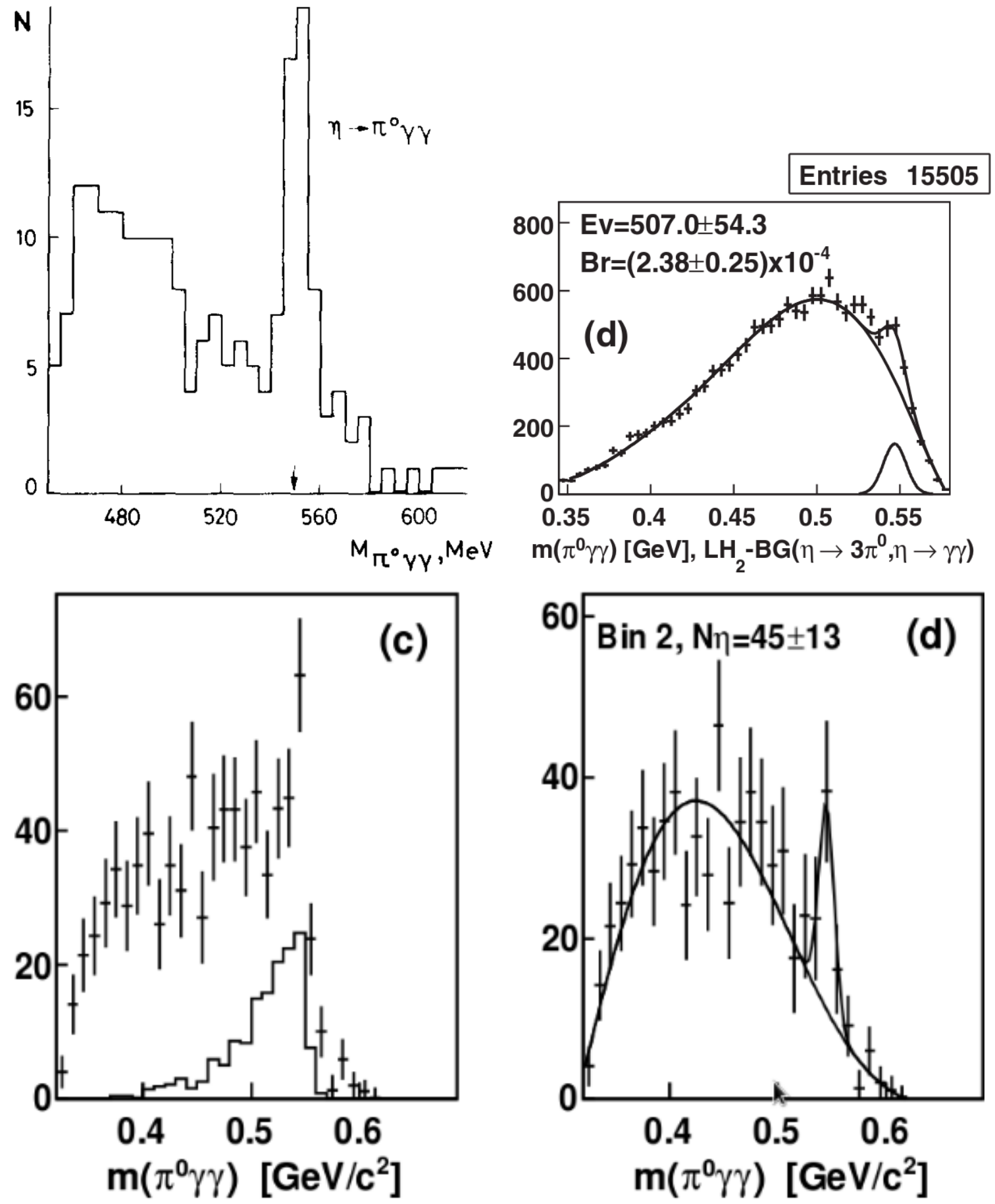

Figure 4: (Top left) Invariant mass distribution for $\pi^{0} \gamma \gamma$ events from [5]. (Top right) Sample $\pi^{0} \gamma \gamma$ invariant mass distribution from [6]. (Bottom row) Sample $\pi^{0} \gamma \gamma$ invariant mass distributions from [7]. 


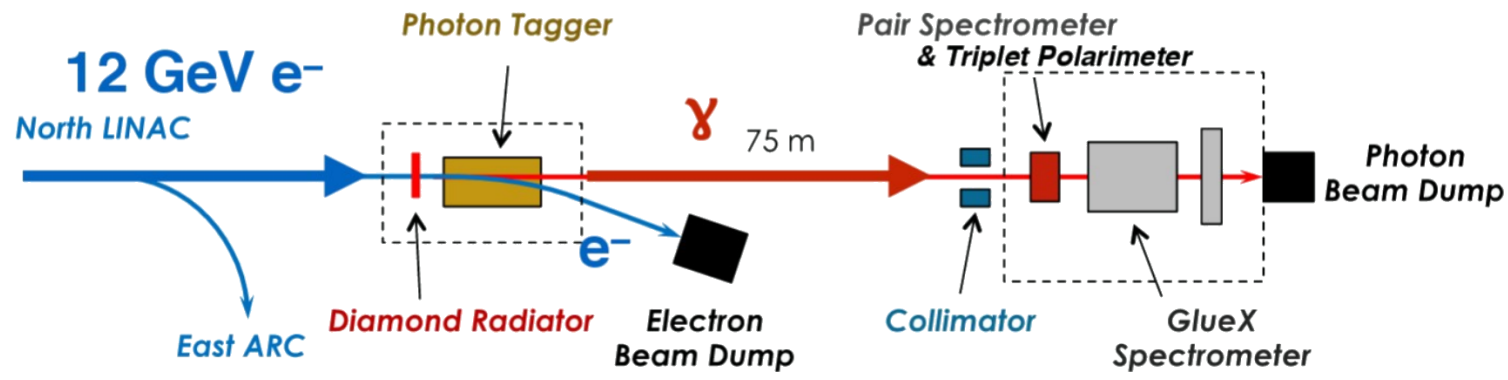

Figure 5: Schematic view of the beam line for the Hall-D complex showing the Tagger hall and the main experimental hall.

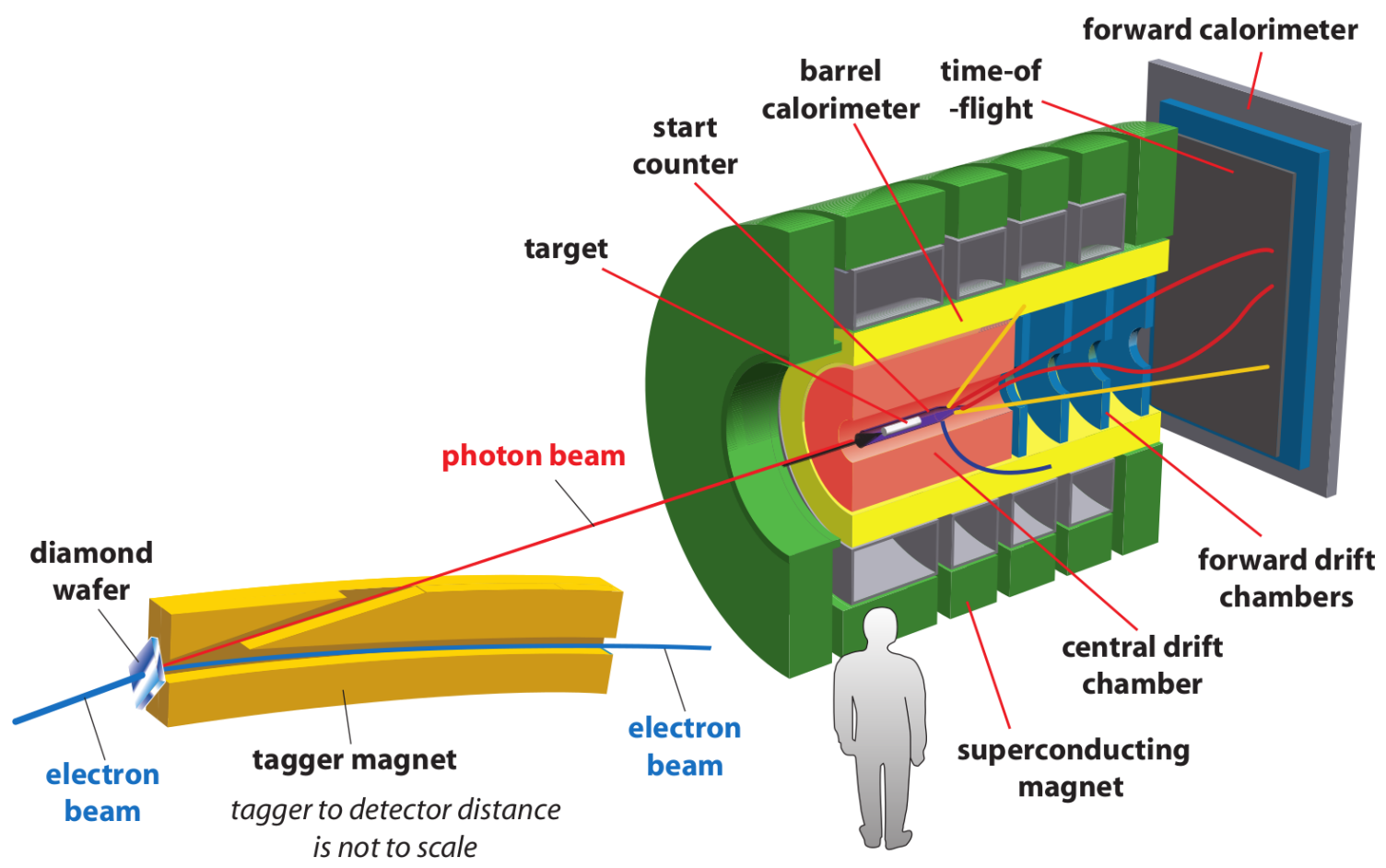

Figure 6: Schematic view of the GlueX detector.

Carlo simulations suggest that a clear peak at the $\eta$ mass will be seen if most of the clusters are in the insert region, as shown in Fig. 10. The expected signal-to-background ratio is about 2.3. Our projected error bars for the decay width are compared to previous measurements in Fig. 11 for 100 days of running. This experimental configuration enables measuring the Dalitz distribution with sufficient statistical precision to distinguish between various model contributions to the line shape, also shown in Fig. 11. The sensitivity for the $B$-boson search is shown in Fig. 12; we estimate a two order-of-magnitude improvement in the upper limit for $B$-boson production in the 150-550 $\mathrm{MeV}$ mass range.

This material is based upon work supported by the U.S. Department of Energy, Office of Science, Office of Nuclear Physics under contract DE-AC05-06OR23177. 
Forward Lead Glass Calorimeter

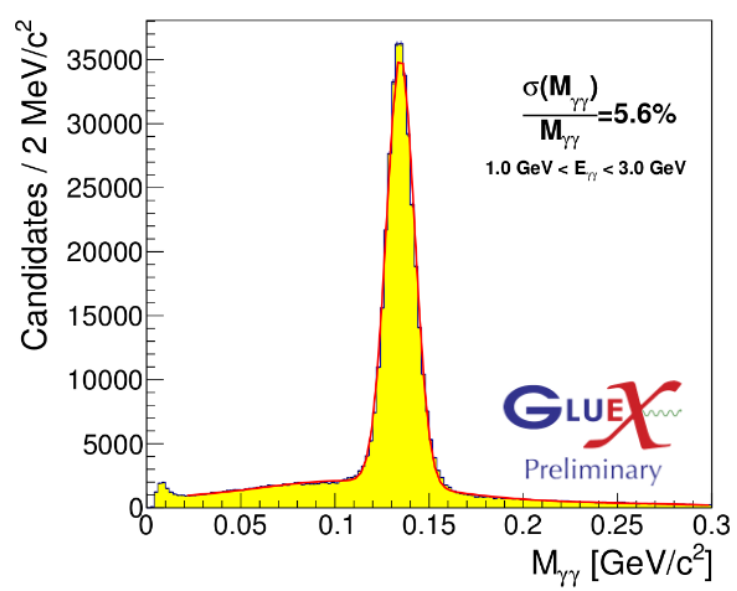

Barrel Lead-Scintillating Fiber Calorimeter

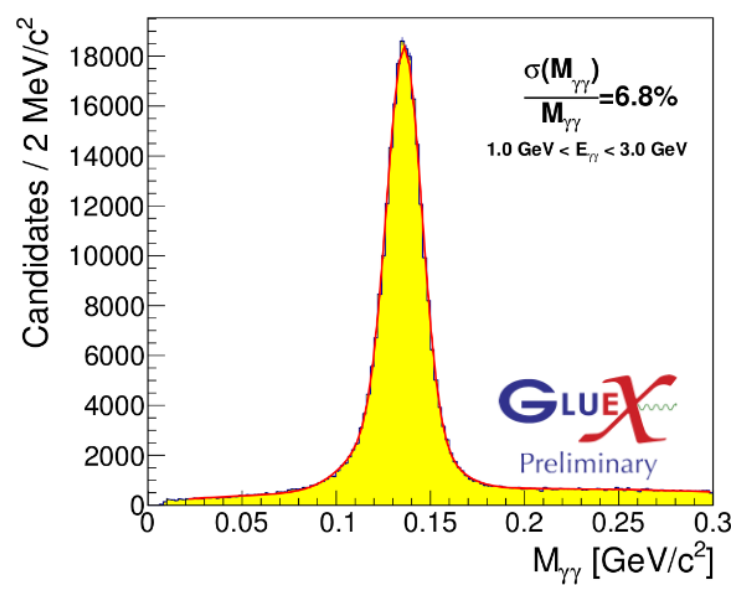

Figure 7: Reconstruction of $\pi^{0} \rightarrow \gamma \gamma$ when both photons are in the FCAL (left) or the BCAL(right).
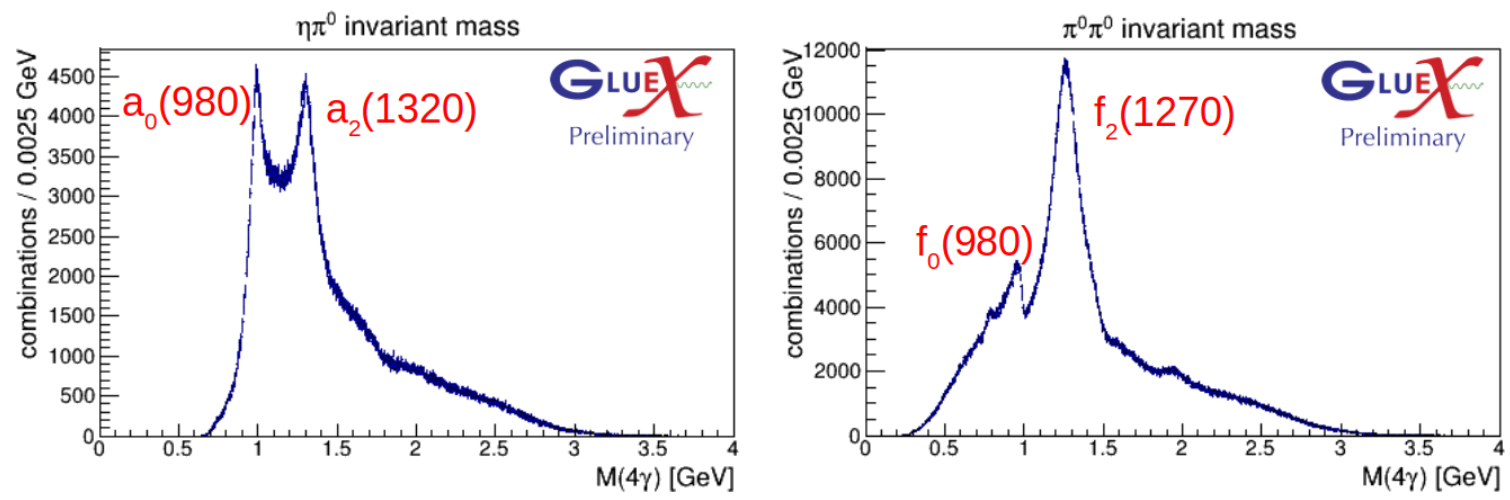

Figure 8: Invariant mass distributions for the reconstruction of four photon events in the $\eta \pi^{0}$ channel (left) and the $\pi^{0} \pi^{0}$ channel (right).

\section{References}

[1] M. Tanabashi et al. [Particle Data Group], Review of Particle Physics, Phys. Rev. D 98, no. 3 (2018) 030001.

[2] L. Ametller, J. Bijnens, A. Bramon and F. Cornet, Chiral perturbation theory for $\eta \rightarrow \pi^{0} \gamma \gamma$, Phys. Lett. B 276 (1992) 185.

[3] E. Oset, J. R. Pelaez and L. Roca, $\eta \rightarrow \pi^{0} \gamma \gamma$ decay within a chiral unitary approach, Phys. Rev. D 67 (2003) 073013.

[4] E. Oset, J. R. Pelaez and L. Roca, $\eta \rightarrow \pi^{0} \gamma \gamma$ decay within a chiral unitary approach revisited, Phys. Rev. D 77 (2008) 073001.

[5] L. G. Landsberg, Electromagnetic Decays of Light Mesons, Phys. Rept. 128 (1985) 301.

[6] S. Prakhov et al., Measurement of the invariant-mass spectrum for the two photons from the $\eta \rightarrow \pi^{0} \gamma \gamma$ decay, Phys. Rev. $C 78$ (2008) 015206.

[7] B. M. K. Nefkens et al. [A2 at MAMI Collaboration], New measurement of the rare decay $\eta \rightarrow \pi^{0} \gamma \gamma$ with the Crystal Ball/TAPS detectors at the Mainz Microtron, Phys. Rev. C 90, no. 2 (2014) 025206. 


\section{$\pi^{0} 2 \gamma$ invariant mass}

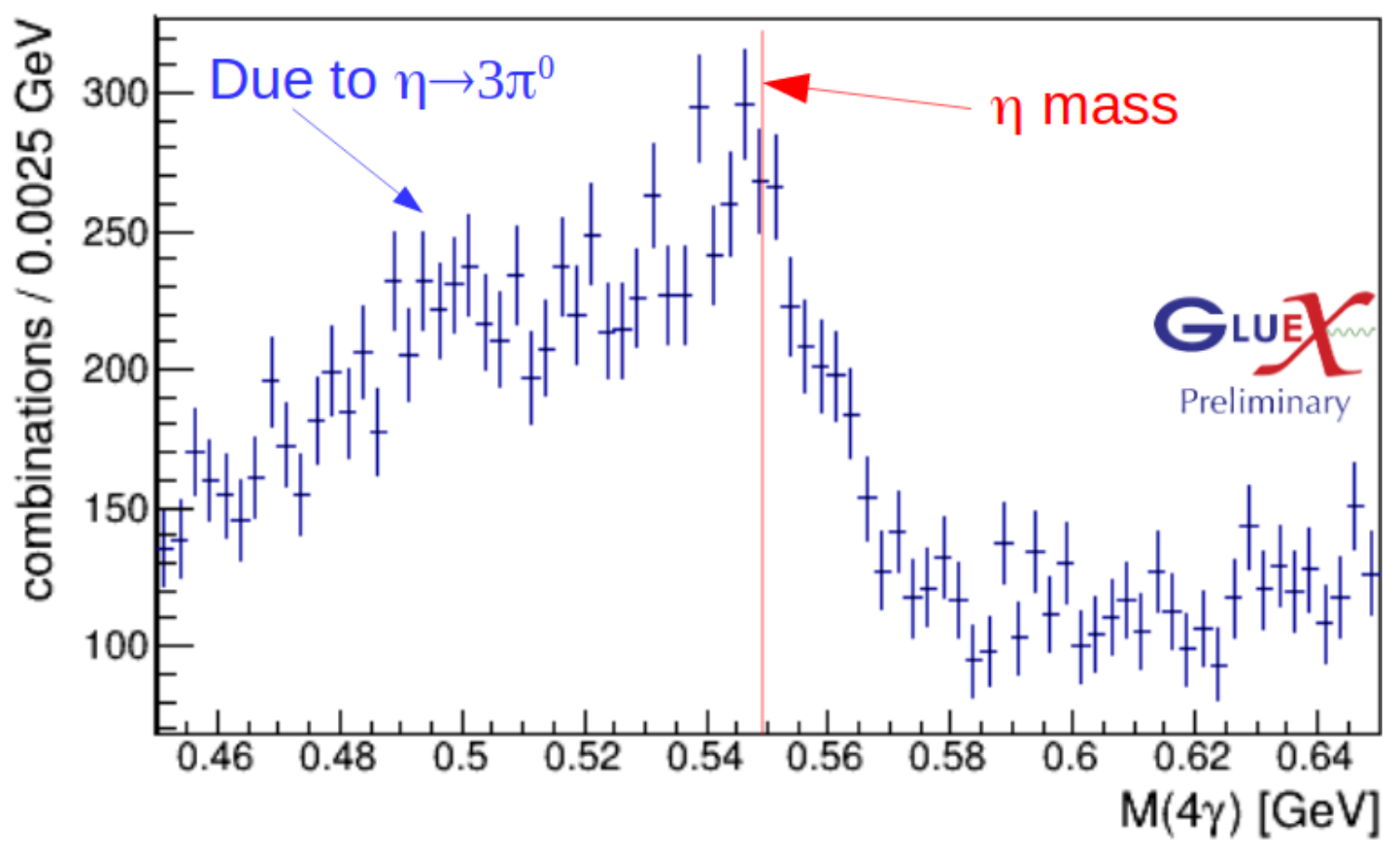

Figure 9: Invariant mass distribution for the $\pi^{0} \gamma \gamma$ channel.

\section{FCAL view from downstream looking upstream}
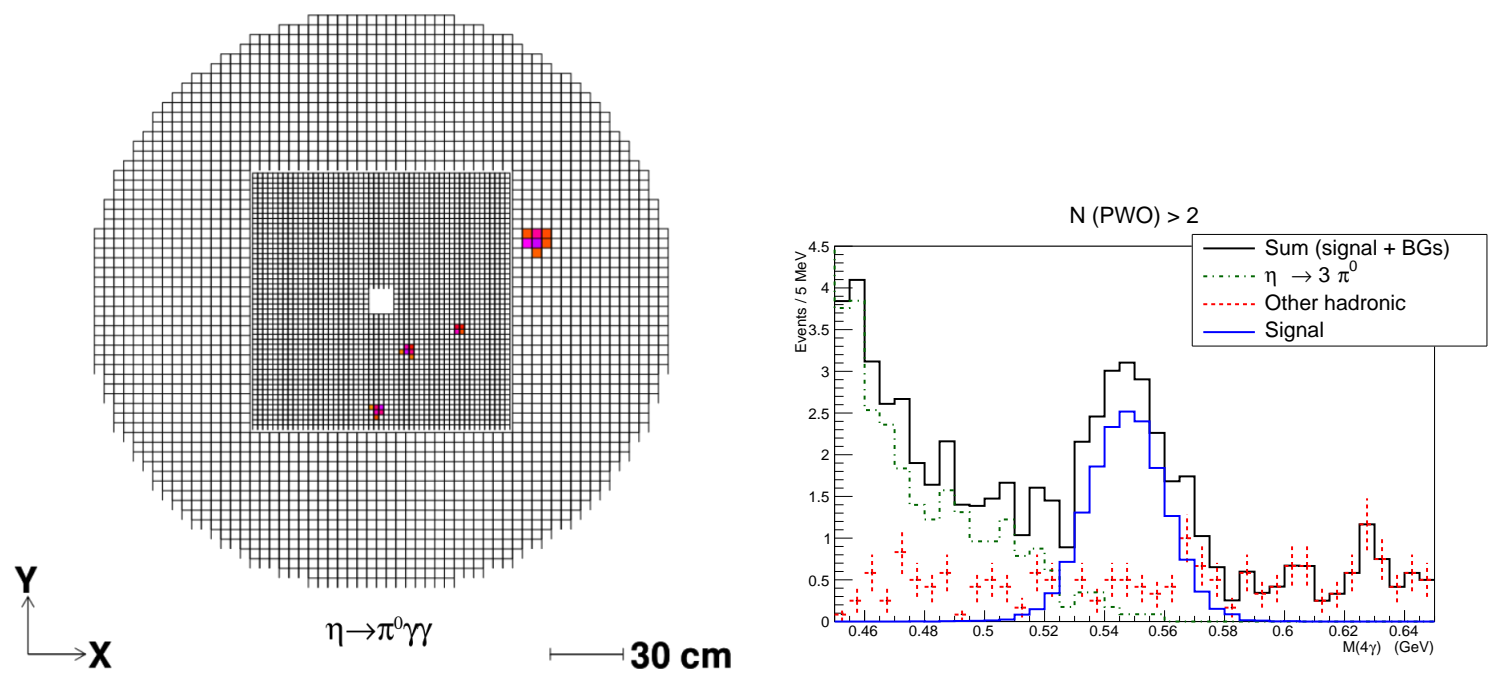

Figure 10: (Left) Front view of a modified FCAL with a $100 \times 100 \mathrm{~cm}^{2}$ insert. Colored boxes indicate showers produced by a simulated $\eta \rightarrow \pi^{0} \gamma \gamma$ event. (Right) Invariant mass of $\pi^{0} \gamma \gamma$ from simulation of the $\pi^{0} \gamma \gamma$ channel along with the dominant background processes for the modified FCAL geometry. We required three out of the four photons to be within the insert. 

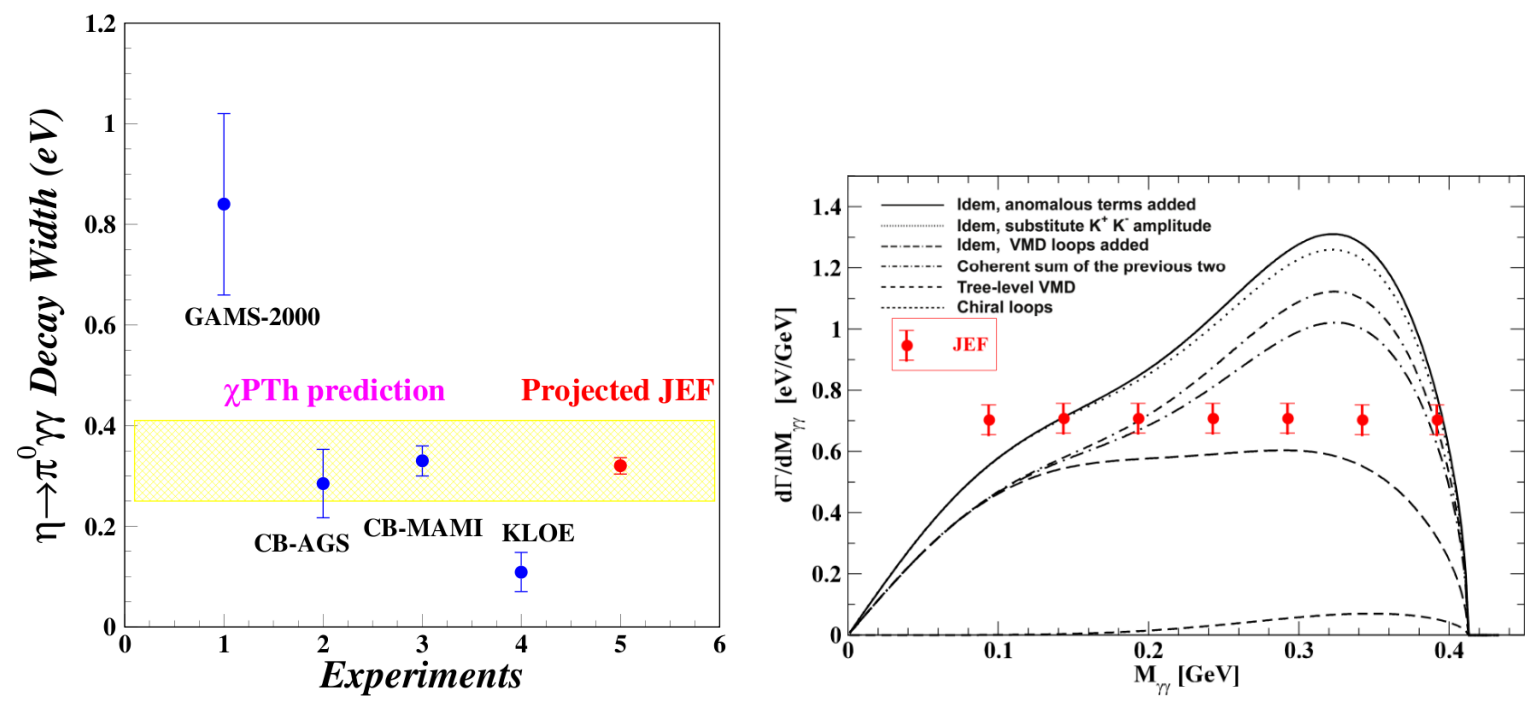

Figure 11: (Left) Comparison of projected statistical uncertainty in the partial width for the $\eta \rightarrow \pi^{0} \gamma \gamma$ channel from the JEF experiment compared to previous measurements: GAMS-2000[5], CB-AGS[6], CBMAMI[7], KLOE[8]. The yellow band corresponds to the model prediction of $\Gamma=0.33 \pm 0.08 \mathrm{eV}$ from [4]. (Right) Projected errors for several mass bins superimposed on the predicted $\gamma \gamma$ mass distribution from [4].

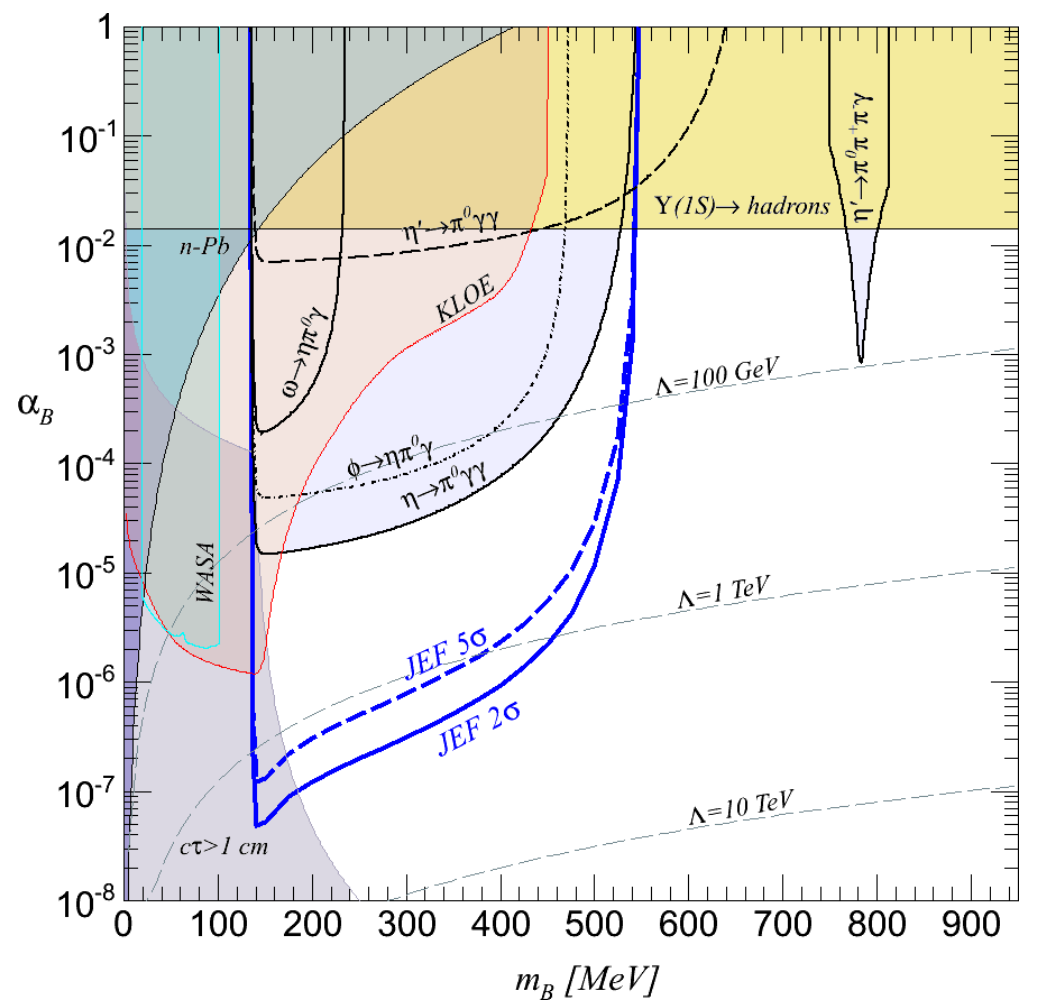

Figure 12: Excluded $B$-boson parameter space with the predicted sensitivity from the JEF experiment superimposed. 
[8] B. Di Micco et al. [KLOE Collaboration], The $\eta \rightarrow \pi^{0} \gamma \gamma, \eta / \eta^{\prime}$ mixing angle and the $\eta$ mass measurement at KLOE, Acta Phys. Slov. 56 (2006) 403.

[9] S. Tulin, New weakly-coupled forces hidden in low-energy QCD, Phys. Rev. D 89, no. 11 (2014) 114008 . 